\title{
Species- and size-related differences in dopamine-like immunoreactive clusters in the brain of Nasonia vitripennis and $N$. giraulti
}

\author{
Jitte Groothuis ${ }^{1} \cdot$ Krista van den Heuvel $^{1,2} \cdot$ Hans M. Smid $^{1}$ (D) \\ Received: 15 November 2018 / Accepted: 24 July 2019 / Published online: 22 August 2019 \\ (C) The Author(s) 2019
}

\begin{abstract}
An extreme reduction in body size has been shown to negatively impact the memory retention level of the parasitic wasp Nasonia vitripennis. In addition, $N$. vitripennis and Nasonia giraulti, closely related parasitic wasps, differ markedly in the number of conditioning trials required to form long-term memory. These differences in memory dynamics may be associated with differences in the dopaminergic neurons in the Nasonia brains. Here, we used dopamine immunoreactivity to identify and count the number of cell bodies in dopaminergic clusters of normal- and small-sized $N$. vitripennis and normal-sized $N$. giraulti. We counted in total a maximum of approximately 160 dopaminergic neurons per brain. These neurons were present in 9 identifiable clusters (D1a, D1b, D2, D3, D4a, D4b, D5, D6 and D7). Our analysis revealed that $N$. giraulti had fewer cells in the D2 and D4a clusters but more in D4b, compared with normal-sized $N$. vitripennis. In addition, we found fewer cells in the D5 and D7 cluster of small-sized $N$. vitripennis compared to normal-sized $N$. vitripennis. A comparison of our findings with the literature on dopaminergic clusters in the fruit fly Drosophila melanogaster and the honey bee Apis mellifera indicates that clusters D2, D3 and D5 may play a role in memory formation in Nasonia wasps. The results from both the species comparison and the size comparison are therefore of high interest and importance for our understanding of the complex intricacies that underlie the memory dynamics of insects.
\end{abstract}

Keywords Dopamine · Brain · Insect · Parasitic wasp · Confocal laser scanning microscopy

\section{Introduction}

Nasonia is a genus of emerging hymenopteran model organisms (Werren and Loehlin 2009) that was recently used in several studies on associative learning (reviewed in Smid and Vet 2016; Liefting et al. 2018). Nasonia wasps parasitize various fly species and lay egg clutches inside the puparium of their fly host, where the wasps develop gregariously. Laying eggs

Electronic supplementary material The online version of this article (https://doi.org/10.1007/s00441-019-03079-7) contains supplementary material, which is available to authorized users.

Hans M. Smid

hansm.smid@wur.nl

1 Laboratory of Entomology, Wageningen University, P.O. Box 16, 6700, AA Wageningen, The Netherlands

2 Present address: Department of Animal Ecology, Netherlands Institute of Ecology (NIOO-KNAW), Wageningen, The Netherlands in a host is a highly rewarding and ecologically relevant experience for the female wasps. As such, it is suitable for use as an appetitive unconditioned stimulus in classical conditioning and can be used to make lasting associations with conditioned stimuli such as odors (Hoedjes et al. 2012). Interestingly, there are strong and distinct differences in the memory dynamics of such conditioning within the Nasonia genus. Nasonia vitripennis wasps consolidate protein synthesis-dependent long-term memory after a single conditioning event, whereas in $N$. giraulti wasps, a single event leads to short-lasting, protein synthesisindependent, memory. Three repeated conditioning events, however, do lead to formation of long-term memory in N. giraulti (Hoedjes and Smid 2014).

In addition to these interspecies differences, different strains also differ in their memory strength (Schurmann et al. 2012; Koppik et al. 2015; Liefting et al. 2018), as do size-related differences within one genotype (van der Woude et al. 2018). Nasonia wasps have a large potential for size plasticity, being able to scale their body size over at least one order of magnitude when forced to deal with strong scramble competition. In 
$N$. vitripennis, this leads to several structural adaptations in the brains of the smallest wasps (Groothuis and Smid 2017). These small-sized $N$. vitripennis individuals have inferior memory retention when compared to larger conspecifics (van der Woude et al. 2018).

Memory dynamics in insect brains are mediated by the modulating neurotransmitters octopamine (OA) and dopamine (DA) (Hammer 1993; Hammer and Menzel 1998; Schwaerzel et al. 2003; Vergoz et al. 2007; Liu et al. 2012; Burke et al. 2012; Das et al. 2016). Whereas previous theories ascribed appetitive conditioning to octopaminergic cells and aversive conditioning to dopaminergic cells (Schwaerzel et al. 2003), it is currently assumed that dopamine in fact plays a role in both aversive as well as appetitive learning and in forgetting (Liu et al. 2012; Burke et al. 2012; Waddell 2013; Rohwedder et al. 2016; Kaun and Rothenfluh 2017; Cognigni et al. 2018). This implies that differences in memory dynamics may relate to the functioning of these modulatory neurotransmitter networks. They may, for example, differ in the number of octopaminergic or dopaminergic cells or in their projections. As a previous description of the octopaminergic network showed no evidence for differences between N. vitripennis and N. giraulti (Haverkamp and Smid 2014), the focus of this study is on the dopaminergic network.

Dopaminergic neurons (DANs) heavily innervate the mushroom body, a multimodal integration center that is thought to be the basis of memory in insects. In Drosophila melanogaster fruit flies, this dopaminergic innervation derives from cells in the protocerebral posterior lateral (PPL1 and PPL2ab) and protocerebral anterior medial (PAM) clusters, with various cells showing discrete topological innervation of specific areas in the mushroom body (Mao and Davis 2009; Waddell 2013; Das et al. 2016). Of these PPL and the PAM clusters, specific DANs (PPL1-MV1, PPL1-MP1 and PAM-M3 cells; Claridge-Chang et al. 2009; Aso et al. 2012) are thought to signal aversion, whereas other DANs in the PAM cluster are assumed to signal reward (Aso et al. 2012; Liu et al. 2012; Burke et al. 2012; Lin et al. 2014). In addition, appetitive memory is associated with a subset of PPL1-MP1 DANs; these cells block appetitive memory retrieval by their tonic dopamine release. However, this activity may be inhibited by signaling of neuropeptide $\mathrm{F}$ (the arthropod analog of mammalian neuropeptide Y) in hungry flies, which improves appetitive memory performance (Krashes et al. 2009).

Thus, the PPL and the PAM cluster are clearly relevant for appetitive memory dynamics in the fruit fly. As their Nasonia homologs may serve similar functions, it is crucial to study this in the context of the observed species- and brain sizespecific differences in associative learning. Here, we investigate differences in numbers and morphology of dopaminergic neurons between normal- and small-sized $N$. vitripennis females and between normal-sized $N$. vitripennis and $N$. giraulti females. First, by analyzing immunoreactivity against dopamine, we will provide a comparison of dopaminergic clusters in the Nasonia brain with the clusters known from the fruit fly (Nässel and Elekes 1992; Mao and Davis 2009) and two hymenopteran species, the honey bee (Tedjakumala et al. 2017) and Trichogramma evanescens (van der Woude and Smid 2017a). This comparison will be based mainly on the comparative location of the clusters within the cell body rind, relative to specific neuropils, as well as the putative projection targets of these clusters. Second, we will compare the number of dopaminergic neurons present in the various clusters between normal-sized $N$. vitripennis and $N$. giraulti (interspecific comparison), as well as between normal- and small-sized $N$. vitripennis (intraspecific comparison). We hypothesize that small-sized $N$. vitripennis females have lower numbers of dopaminergic neurons in the brain than normal-sized females. If such differences are correlated to the variation in memory dynamics after oviposition-based appetitive learning, we expect to find them in the same clusters in the interspecific comparison as in the intraspecific comparison.

\section{Materials and methods}

\section{Insects}

Isogenic strains of $N$. vitripennis (AsymCx) and $N$. giraulti $(\mathrm{RV} 2 \mathrm{x}(\mathrm{U}))$ were used. Both strains have previously been used to study memory formation (Hoedjes and Smid 2014). They have a sequenced genome and genetic tools have been developed for these strains (Werren et al. 2010; Rago et al. 2016). Rearing was performed and differences in body size were induced by varying levels of scramble competition as described previously (Groothuis and Smid 2017). In short, normal-sized wasps were obtained by rearing on Calliphora vomitoria pupae (Kreikamp BV, Hoevelaken, The Netherlands) at a ratio of 10 female wasps to 20 pupae. Small-sized $N$. vitripennis were obtained by rearing at a ratio of 50:5. Females were allowed to oviposit for $24 \mathrm{~h}$ in both cases. For experiments, female wasps were collected within 1 day of eclosion and kept in vials with access to water and honey. The 50:5 rearing shows a large variation in female body size; for the experiments, only small-sized $N$. vitripennis wasps with a head width under $500 \mu \mathrm{m}$ were used. The heads of normal-sized $N$. vitripennis and $N$. giraulti were not measured but generally have head widths of approximately $750 \mu \mathrm{m}$ (Groothuis and Smid 2017).

\section{Immunohistochemistry}

Cold-sedated wasps were decapitated in ice-cold fixative: one part 25\% glutaraldehyde (Sigma) and three parts saturated picric acid with $0.1 \%$ acetic acid (GPA). To allow penetration 
of the fixative, the antennae were removed and small openings made in the eyes. Heads were fixed for $4 \mathrm{~h}$ at room temperature (RT) and then washed overnight in $70 \% \mathrm{EtOH}$ prior to dissection of the brains in $70 \%$ EtOH.

After dissection, brains were dehydrated in a graded series of EtOH concentrations (70-90-96-100-100\%, 50\% $\mathrm{EtOH} / \mathrm{xylene}, 2 \mathrm{~min}$ each), degreased in $100 \%$ xylene and rehydrated to phosphate-buffered saline (PBS; Oxoid, Dulbecco "A" tablets) (via 50\% EtOH/xylene, 100-96-9080-70-50-30\% EtOH). To reduce oxidized dopamine, brains were incubated for $20 \mathrm{~min}$ in freshly prepared $1 \%$ sodium borohydrate in PBS. After rinsing four times for $5 \mathrm{~min}$ and 3 times for $15 \mathrm{~min}$ in PBS, the brains were treated with $5 \mathrm{mg} / \mathrm{mL}$ collagenase (Sigma) in PBS for $1 \mathrm{~h}$ at RT, improving the permeability of the tissue. Subsequently, the brains were rinsed four times for $5 \mathrm{~min}$ in PBS containing $0.5 \%$ Triton$\mathrm{X} 100$ (PBS-T) and preincubated in 10\% normal goat serum (NGS; Dako, Glostrup, Denmark) in PBS-T (PBS-T-NGS) for $1 \mathrm{~h}$ at RT. The primary mouse anti-dopamine antiserum (Millipore, catalog no. MAB5300, RRID:AB_94817) was incubated overnight at RT at dilutions of 1:200 for normal-sized brains or 1:66.7 for small brains in PBS-T-NGS. After five 30min washes in PBS-T, a secondary rabbit anti-mouse antibody (Dako, catalog no. Z0259, RRID:AB_2532147) was applied at a 1:200 dilution in PBS-T-NG for $3 \mathrm{~h}$ at room temperature. Finally, following four 30-min washes in PBS-T, a tertiary antiserum of goat anti-rabbit antibodies linked to AlexaFluor® 488 (Jackson ImmunoResearch Labs, catalog no. 115-545-003, RRID:AB_2338840) was used at a dilution of $1: 100$ together with propidium iodide at a dilution of $1: 200$ in PBS-T-NGS overnight at $4{ }^{\circ} \mathrm{C}$. In pilot experiments, this triple antibody approach was superior to dual antibody labeling in providing a suitable signal-to-noise ratio when imaging.

Whole-mount preparations were made by washing four times for $30 \mathrm{~min}$ in PBS-T and dehydration and degreasing to xylenes (via 30-50-70-80-90-96-100-100\% EtOH, 50\% $\mathrm{EtOH} / x y l e n e, 2 \mathrm{~min}$ each), followed by mounting on a microscope slide in a drop of DPX mounting medium (Sigma) under a No. 1 cover slip.

\section{Antiserum specificity}

The monoclonal antibody was raised against a glutaraldehyde conjugate of dopamine and bovine serum albumin (DA-GBSA), as specified by the manufacturer. In the brains, dopamine will therefore only be detected when crosslinked by glutaraldehyde and this chemical is therefore an essential component of the fixative solution. We used the term dopamine-like immunoreactivity (DA-L-IR) to indicate this fact in our descriptions below. Cross-reactivity of the mouse anti-dopamine antibody was determined as specified by the manufacturer: DA-G-BSA 1; L-DOPA-G-BSA 1:10,000; Tyrosine-G-BSA 1:36,000; Tyramine-G-BSA 1:> 50,000;
Noradrenaline-G-BSA 1:> 50,000; OA-G-BSA 1:> 50,000; Adrenaline-G-BSA 1:>50,000; DA 1:>50,000. We performed additional control experiments by using preparations without primary antisera. These did not reveal any immunolabeling. In addition, in the Electronic Supplementary Material, we show a high similarity between this anti-dopamine labeling and an anti-tyrosine hydroxylase labeling that became available after this study was completed.

\section{Microscopy}

Preparations were scanned with a Zeiss LSM510 microscope equipped with a $\times 40$ oil immersion objective (Plan-Neofluar $\times 40 / 1.3$ ) and $\times 63$ oil immersion objective (Plan-Apochromat $\times 63 / 1.4$ ). AlexaFluor ${ }^{\circledR} 488$ staining was imaged using the 488-nm line of an Argon laser with a 505- to 550-nm BP filter; the propidium iodide nuclear counterstain was imaged using the same excitation wavelength but with a 560-nm LP filter. Scanning was performed at 8 bit with a resolution of $1024 \times$ 1024 , with voxel dimensions ranging from $0.36 \times 0.36 \times 2$ to $0.14 \times 0.14 \times 0.38 \mu \mathrm{m}$.

\section{Nomenclature}

Nomenclature of neuropils in the $N$. vitripennis and $N$. giraulti brains was used as described previously (Groothuis and Smid 2017) and according to standardized nomenclature (Ito et al. 2014). Orientation and positions in the brain are given using the body axis. DA-L-IR clusters were numbered from anterior to posterior, as was done previously in the honey bee Apis mellifera (Schürmann et al. 1989; Tedjakumala et al. 2017), the parasitic wasp Trichogramma evanescens (van der Woude and Smid 2017a) and for octopaminergic clusters in N. vitripennis and N. giraulti (Haverkamp and Smid 2014). When clusters were found at comparable depths, preference was given to dorsal clusters. As there is no clear boundary of the fusion between the supra- and suboesophageal ganglia in Nasonia brains, we did not consider this division in naming the DA-L clusters.

\section{Neuron analysis}

The selection of suitable brains for analysis and the counting of DA-L-IR neurons was performed blinded to the identity (species or body size) of the brain sample by randomization of files after image acquisition. The initial sample consisted of 51 brains, based on a visual assessment of staining quality; during analysis, several more samples were removed, bringing the total to 38 analyzed brains. This reduction was based on the visibility of clusters of dopaminergic neurons in at least one of the two hemispheres. Visibility could be impaired by torn tissue, insufficient signal-to-noise ratio, or occlusion by non-brain particles. The number of cell bodies was only 
analyzed in brains in which individual somata within DA-LIR clusters were clearly visible and the best-stained hemisphere was selected for analysis of individual clusters. Cell bodies were counted by means of image segmentation in Amira 5.4 (Visage Imaging, Berlin, Germany; currently distributed by Thermo Fisher Scientific), assigning each cluster a separate LabelField and each cell an individual Material. During this process, the propidium iodide counterstain could be used for orientation but is not used for other purposes. Due to tissue damage, not all identified clusters could be counted in every sample. After segmentation, results were exported with the MaterialStatistics module and collected in a spreadsheet for further analysis.

\section{Statistics}

We compared the number of DA-L cell bodies for all identified clusters separately and in a pairwise fashion for the following combinations: $N$. vitripennis (normal-sized) $\times$ $N$. giraulti (normal-sized) and $N$. vitripennis (normal-sized) $\times$ $N$. vitripennis (small-sized).

Comparison between cluster-species combinations was analyzed with a non-parametric Mann-Whitney-Wilcoxon test $(\alpha=0.05)$. All statistical analyses were performed in $\mathrm{R}$ version 3.3.2.

\section{Results}

\section{Overall tissue and labeling quality}

The triple-labeling protocol ensured sufficient staining quality in most brains. However, the glutaraldehyde fixation of the brains in the head capsule before dissection (required for the conjugation and subsequent detection of the antigen) caused relatively fragile brains that are difficult to dissect from the head capsule, as the tissue is more brittle than after formaldehyde fixation or in unfixed dissections. In many preparations, this led to a damaged cell body rind or loss of tissue, mainly of the optic lobes. In addition, the rind at the anterior and the central sides of the brain was found to be especially fragile, which on occasion caused either damage to or removal of the rind, or an occlusion of relevant clusters by non-neural and strongly autofluorescent tissues. Those brains that were not suitable for analysis were removed from the dataset as described below.

Prior to the blinded DA-L-IR analysis, 51 brains were selected (blind to their identity) from the available preparations; of those, 20 were normal-sized $N$. vitripennis, 15 small-sized $N$. vitripennis and $26 N$. giraulti. These numbers were reduced to 15,9 and 14 brains, respectively, after closer examination of the cell clusters during the analysis. The low number of intact small-sized $N$. vitripennis brains was striking in particular and was probably due to the added brittleness of glutaraldehyde fixation, as these brains proved more resilient when dissected as unfixed tissue (Groothuis and Smid 2017).

\section{Dopaminergic clusters in the $\mathrm{N}$. vitripennis brain}

Our analysis of DA-L-IR in N. vitripennis and $N$. giraulti resulted in the identification of nine uniquely identifiable cell groups, two of which we consider a subdivision of another cluster, see Figs. 1, 2 and 3(a) for an overview. Cell numbers are given as average count $\pm \mathrm{SD}(N=$ number of hemispheres analyzed) and based on normal-sized $N$. vitripennis:

Cluster D1a (Fig. 3b) is the most anterior cell cluster, located laterally of the anterior optic tubercle. In all cases, it comprises at least two large and heavily stained cell bodies; in some brains, several additional cells can be identified near this location, which we considered as belonging to Dla. On average, we counted $3.7 \pm 0.7(N=13)$ D1 cells in the $N$. vitripennis brain. No clear projection is observed.

Cluster D1b (Fig. 3c) is a small cluster that always consists of 2 small DA-L cells. It lies at the same level and laterality as cluster D1 but more ventrally. This cluster is only found in a minority of preparations $(N=4)$. No clear projection is observed.

Cluster D2 (Fig. 3d) is the largest cluster, containing $59.6 \pm$ 11.1 cells $(N=13)$. The largest concentration of DA-L cells in this cluster is found at the midline, approximately halfway between the antennal lobe and the distal end of the vertical lobe of the mushroom body, from which more cell bodies are scattered laterally towards and surrounding the anterior optic tubercle. Cell bodies may lie more dorsally or ventrally to the tubercle in individual brains. The neurilemma at the midline forms a clear boundary between hemispheres, so the cluster cannot be confused for an unpaired cluster. In several brains, we observed a gap between the population of more laterally scattered cells and the concentrated mass near the midline, indicating that cluster D2 can probably be subdivided into 2 subclusters. The majority of brains, however, did not show this, so all cell bodies were grouped into cluster D2 in this study. Clear projections into the nearby neuropil are rarely observed and quickly become untraceable.

Cluster D3 (Fig. 3e) lies at the posterior side of the protocerebrum, where it surrounds the proximal end of the mushroom body pedunculus, wedged between the neuropils of the protocerebrum and mushroom body calyx. With $42.5 \pm$ $7.1(N=11)$ cell bodies, it is the second largest cluster. Most cells are found at the anterior and lateral side of the pedunculus. Results from a minority of preparations hint at a gap between the anterior and lateral groupings of cells in this cluster, suggesting that, like in cluster D2, a subdivision of cluster D3 may be warranted. Many of the cells in this cluster contribute to a heavily stained fiber bundle that crosses the pedunculus anteriorly and has several branching points. Some 
a

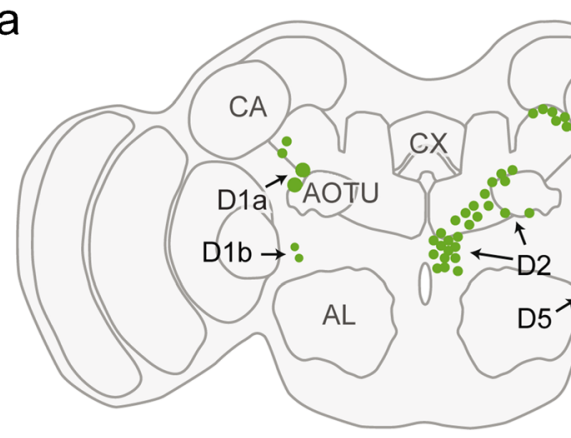

b

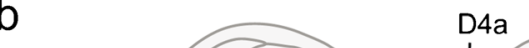

C

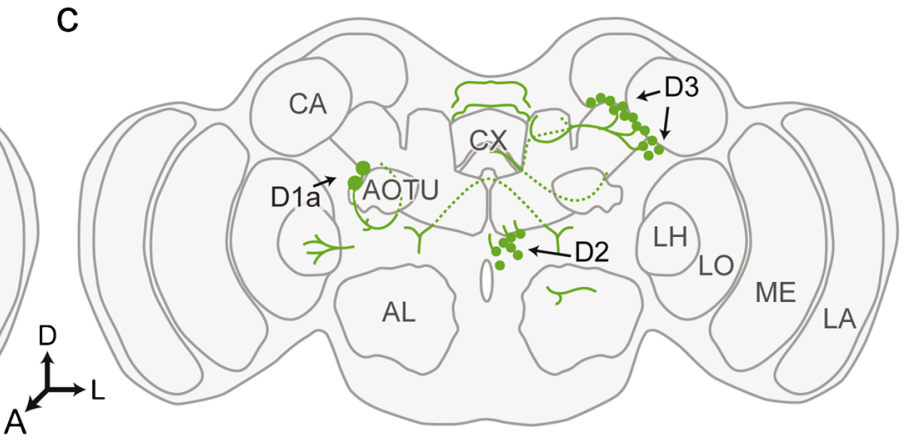

d

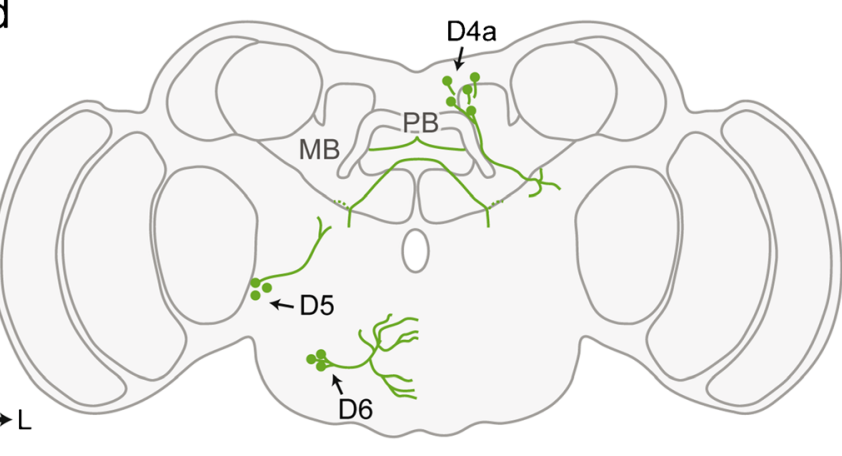

Fig. 1 Schematic representation of DA-L-IR cluster locations (a anterior; b posterior) and major arborizations (c anterior; $\mathbf{d}$ posterior) in the brains of $N$. vitripennis and $N$. giraulti. Locations and arborizations are only drawn for one brain hemisphere. The antennal lobes are scaled to $80 \%$ of the original size to reveal the subesophageal zone neuropils. A anterior,
AL antennal lobe, AOTU anterior optic tubercle, CA calyx, CX central complex, D dorsal, L lateral, LH lateral horn, LO lobula, MB mushroom body, ME medulla, $\mathrm{P}$ posterior, $\mathrm{PB}$ protocerebral bridge (part of CX). Arrows point to clusters

Cluster D4b (Fig. 3g) lies at the most posterior edge of the cell body rind, at a similar dorsal level as cluster D4a. It consists of $5.9 \pm 1.3(N=8)$ slightly scattered cell bodies. No clear projection is observed.

Cluster D5 (Fig. 3h) consists of several $(3.75 \pm 1.2$ cell bodies, $N=8$ ) cells posterior in the brain, located in the thin layer of the cell body rind between the neuropil of the in the dorsal cell body rind. It projects ventrally into the posterior side of the brain.

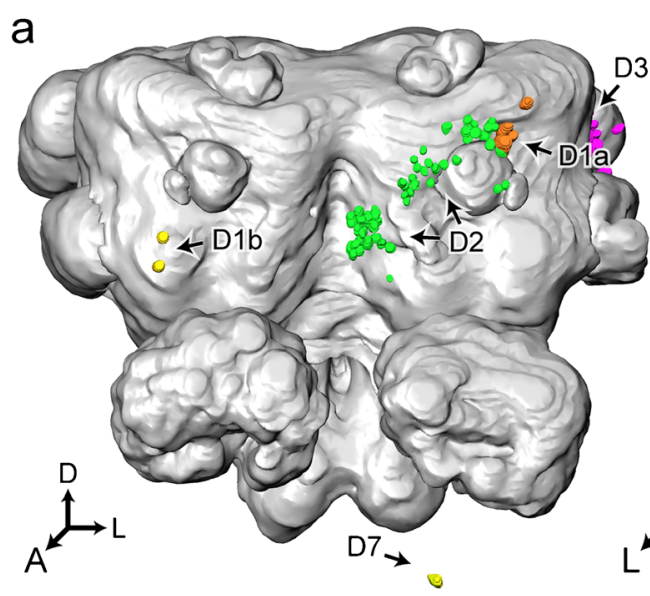

Fig. 2 Location of DA-L-IR somata in the cell body rind (not shown) of a single $N$. vitripennis brain seen from an anterior (a), lateral (b) and posterior (c) perspective. The combined neuropil of the brain is shown in gray. Each cluster is shown with randomized colors in only one of the
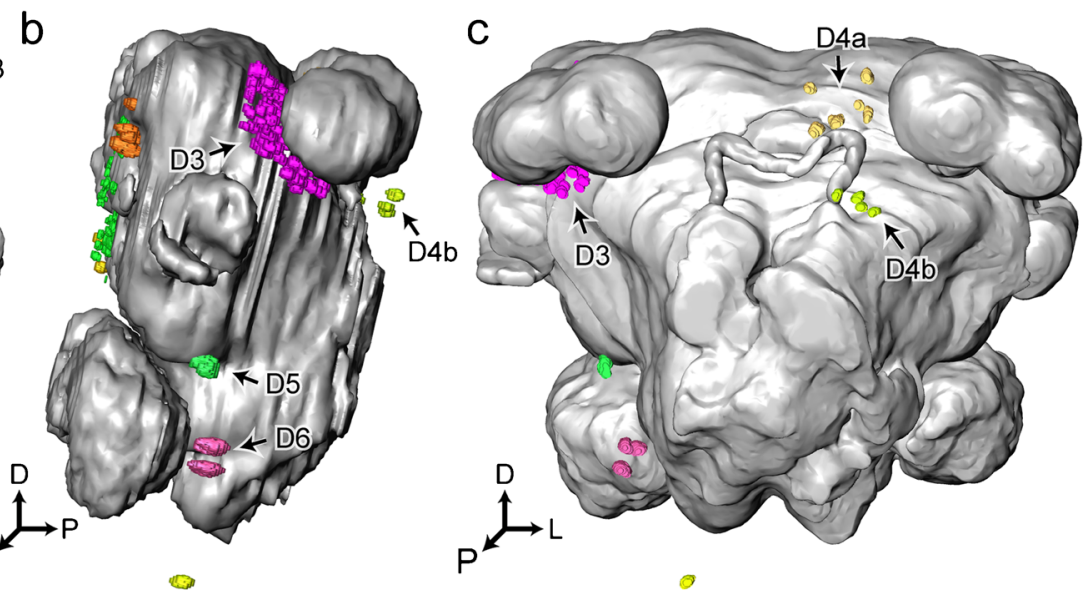

hemispheres. Note that the optic lobes are omitted from the neuropil reconstruction. A anterior, D dorsal, L lateral, $\mathrm{P}$ posterior. Arrows point to clusters 

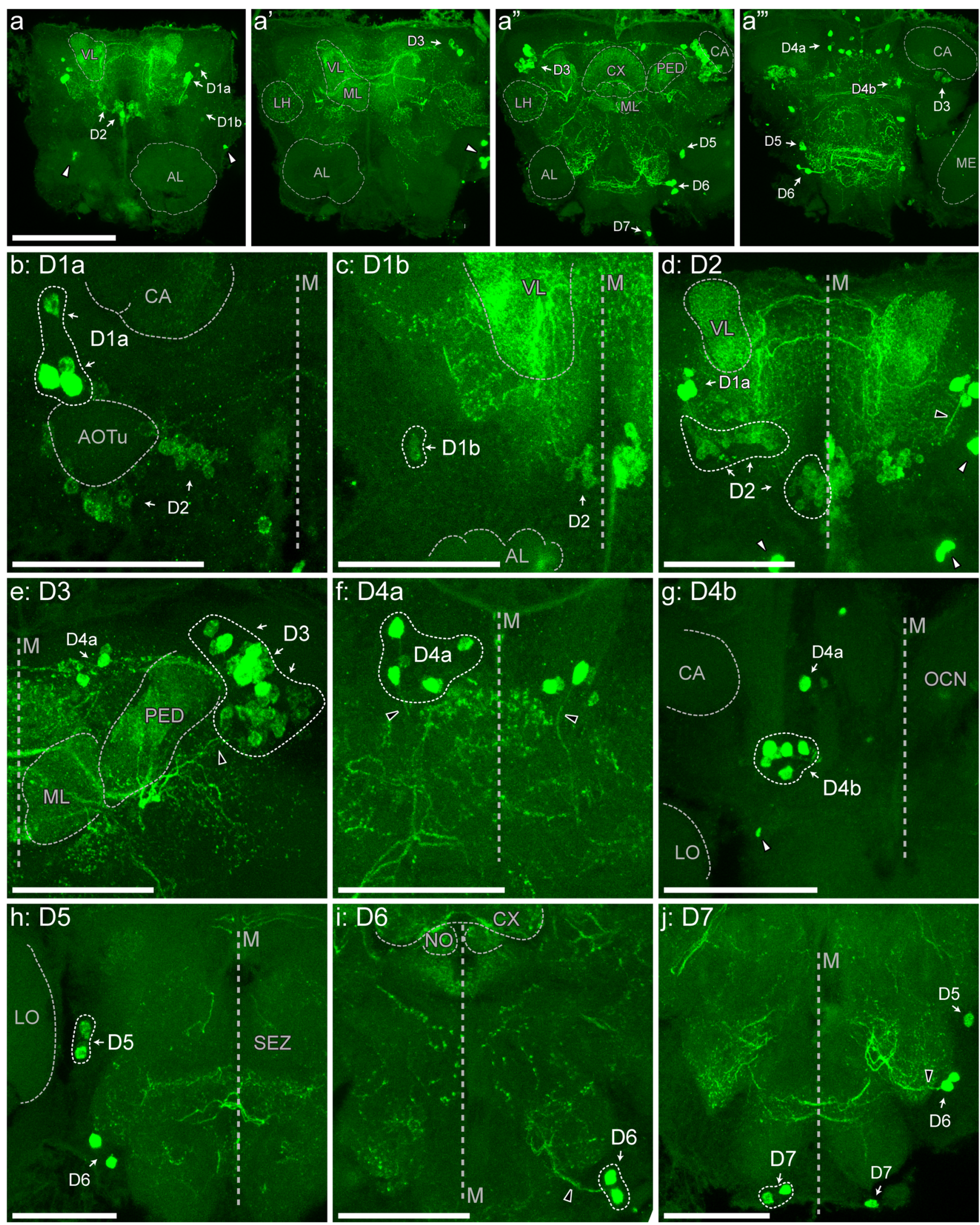

central brain and the optic lobe lobula. No clear projection is observed.
Cluster D6 (Fig. 3i) always consists of $3(N=8)$ large cell bodies, ventrally in the brain, flanking the subesophageal 
Fig. 3 DA-L-IR cell body clusters in the $N$. vitripennis brain, with visible neuropils and midline $(\mathrm{M})$ annotated. Each panel is oriented dorsal side up and contains a maximum intensity projection of varying thickness, highlighting a single cluster. a An overview of DA-L-IR in a single brain; four $30-\mu \mathrm{m}$-thick maximum intensity projections through the brain from anterior (a) to posterior (a"'), annotations only in one of the hemispheres. b Cluster D1a. c Cluster D1b. d Cluster D2. e Cluster D3. f Cluster D4a. g Cluster D4b. h Cluster D5. i Cluster D6. j Cluster D7. AL antennal lobe, AOTU anterior optic tubercle, CA calyx (MB), CBL central body, lower division (CX), CBU central body, upper division (CX), CX central complex, $\mathrm{LH}$ lateral horn, $\mathrm{LO}$ lobula, $\mathrm{M}$ midline, $\mathrm{MB}$ mushroom body, $\mathrm{ME}$ medulla, ML medial lobe (MB), NO nodulus (CX), OCN ocellar neuropils, PED pedunculus (MB), SEZ subesophageal zone. Arrows point to cell clusters; white arrowheads point to staining artifacts; black arrowheads point to cell body fibers. Scale bars depict $100 \mu \mathrm{m}$ in panels $\mathbf{a}-\mathbf{a} ",, 50 \mu \mathrm{m}$ in panels $\mathbf{b}-\mathbf{j}$

zone. Its cell body fibers are often heavily stained and project to nearby neuropils in the SEZ and potentially to protocerebral neuropils as well.

Cluster D7 (Fig. 3j) lies at the most ventral edge of the subesophageal zone and consists of $2(N=3)$ neurons. The cell body rind at its location is easily damaged and this cluster is often lost in preparations. A thin projection is sometimes observed and subsequently lost in the heavily innervated SEZ.

\section{Dopaminergic innervation in the N. vitripennis brain}

Many neuropils in the central brain are highly innervated by DA-L-IR fibers. Due to their abundance, as well as the varicose nature of the immunofluorescence, these fibers cannot be traced back to the cell body clusters from which they project. In a limited number of cases, however, cell body fibers (also described as primary neurites) can be observed in samples. Here, we describe the innervation of several landmark neuropils and, if possible, the location of DA-L-IR fibers projecting towards these neuropils:

The antennal lobe (Fig. 4a) is largely devoid of DA-L-IR innervation, though in most preparations, a low number of small fibers was observed in the non-glomerular antennal lobe hub.

We found no evidence of DA-L-IR innervation in the optic lobes (Fig. 4b).

The anterior optic tubercle (Fig. 4c) contains many varicose DA-L-IR terminals. Although the AOTU is surrounded by cell bodies of cluster D1a and D2, its innervation does not originate from these clusters.

The mushroom body (Fig. $4 \mathrm{~d}-\mathrm{g}$ ) is strongly innervated in the peduncle (Fig. 4d), medial lobe (Fig. 4e) and vertical lobe (Fig. 4f). We have, however, not reliably observed innervation in the calyx (Fig. 4g).

The central complex (Fig. 4h, i) is strongly innervated in the upper division of the central body and the noduli and less in the lower division of the central body (Fig. 4h). No innervation was observed in the protocerebral bridge (Fig. 4i).
The lateral horn (Fig. 4j) is innervated with many varicose terminals. The projections entering the lateral horn were rarely visible and could not be reliably traced back to cell bodies.

The subesophageal zone (Fig. 4k) is home to an extensively branched DA-L-IR arborization that is strongly stained in most preparations. It originates from cluster D6, though input from other clusters, in particular D7, appears likely and cannot be disproven.

\section{Species-specific differences in dopaminergic neurons}

Nasonia vitripennis and $N$. giraulti did not show any outwardly recognizable morphological differences in their neuropils, nor did we note differences in the location or projection patterns of the DA-L-IR clusters. Most clusters showed a similar number of cells counted per cluster for the two Nasonia species (Table 1, Fig. 5a, b) and the maximum number of DA-LIR cells observed in a single brain was also similar (N. vitripennis: 143. N. giraulti: 138). However, we found a significant difference indicating fewer cells in the D2 cluster of $N$. giraulti than in that of $N$. vitripennis (Wilcoxon, $W=$ $34.5, p=0.011)$. In addition, we found fewer cells for $N$. giraulti than for $N$. vitripennis in the D4a cluster (Wilcoxon, $W=34.5, p=0.013$ ) but more in the D4b cluster (Wilcoxon, $W=40, p=0.038$ ).

\section{Effect of brain size on numbers of dopaminergic cell bodies in N. vitripennis}

Despite the large difference in total brain size between normalsized and small-sized N. vitripennis (Groothuis and Smid 2017), the maximum number of DA-L-IR cell bodies counted in a single brain was similar in these groups ( $N$. vitripennis (normal): 143. N. vitripennis (small): 138). We did find several differences in numbers of cells per cluster (Fig. 6a, b). Our analysis indicates fewer cells in the D5 cluster of small-sized $N$. vitripennis (Wilcoxon, $W=52.5, p=0.027$ ), as well as fewer cells in $\mathrm{D} 7$ (Wilcoxon, $W=12, p=0.025$ ). However, this D7 cluster could be counted in only three and four brains, for normal-sized and small-sized $N$. vitripennis, respectively. In addition, we found a marginally significant lower number of D1a cells in small- versus normal-sized $N$. vitripennis (Wilcoxon, $W=85.5, p=0.0502$ ).

\section{Discussion and conclusion}

Previous studies have identified differences in memory dynamics between Nasonia species, as well as $N$. vitripennis of different sizes. Understanding the factors that underlie these differences may lead to novel insights in the evolution variation in memory dynamics (Smid and Vet 2016). To that end, this study aimed to describe the dopaminergic neurons and 


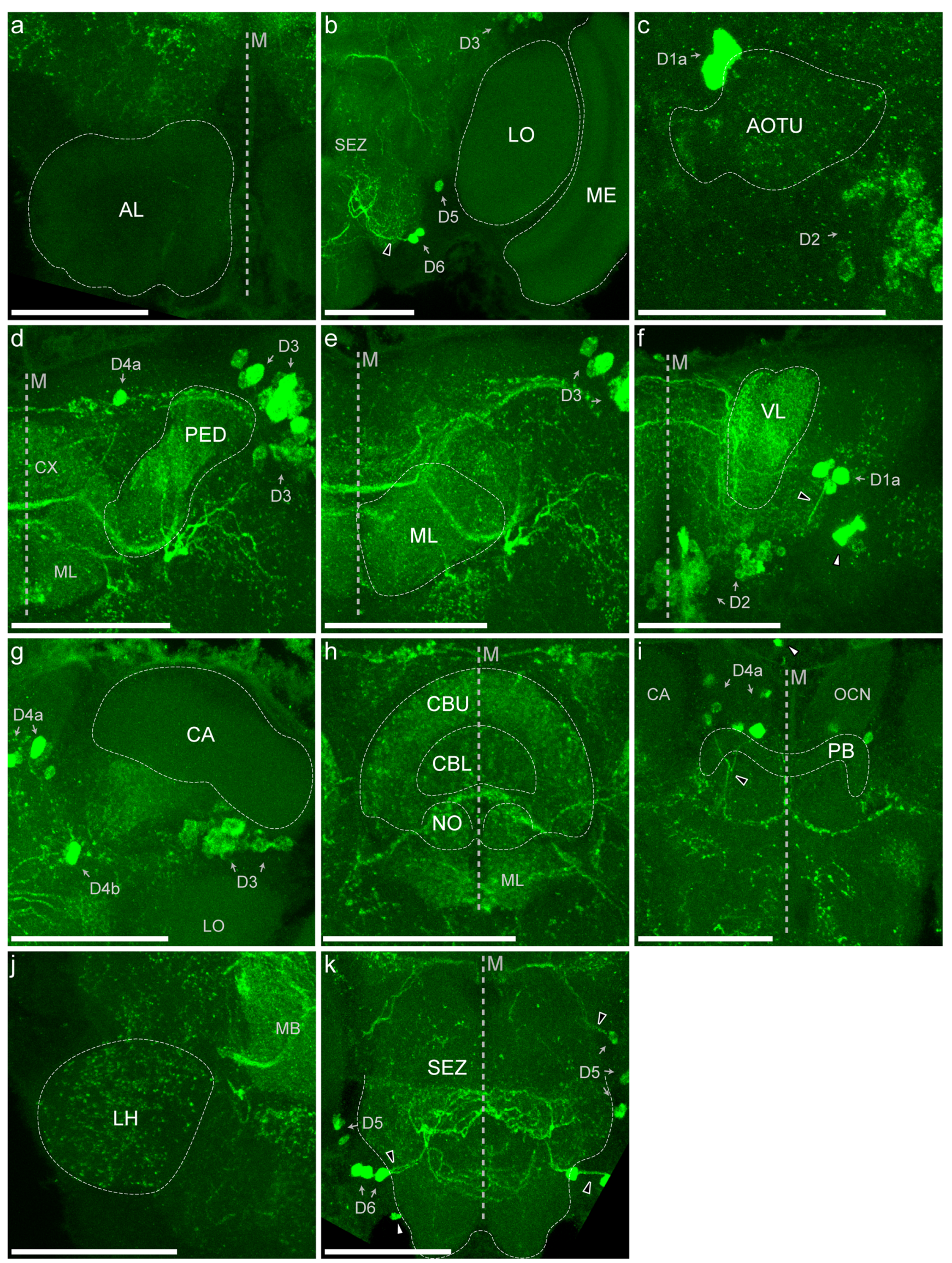


Fig. 4 DA-L-IR projections in the N. vitripennis brain, focusing on innervations of landmark neuropils, with visible clusters and midline (M) annotated. Each panel is oriented dorsal side up and contains a maximum intensity projection of varying thickness, highlighting a single neuropil. When visible, (parts of) the clusters are annotated. a Antennal lobe. b Optic lobe (lobula and medulla). c Anterior optic tubercle. d-g Mushroom body peduncle, medial lobe, vertical lobe and calyx. h Central complex (upper and lower division of the central body and noduli). $\mathbf{i}$ Protocerebral bridge. $\mathbf{j}$ Lateral horn. k Subesophageal zone. AL antennal lobe, AOTU anterior optic tubercle, CA calyx (MB), CBL central body, lower division (CX), CBU central body, upper division (CX), CX central complex, LH lateral horn, LO lobula, M midline, MB mushroom body, ME medulla, ML medial lobe (MB), NO nodulus (CX), OCN ocellar neuropils, PB protocerebral bridge (CX), PED pedunculus (MB), SEZ subesophageal zone. Arrows point to cell clusters; white arrowheads point to staining artifacts; black arrowheads point to cell body fibers. Scale bars depict $50 \mu \mathrm{m}$

find potential correlations between the observed differences in memory dynamics and the morphology of the dopaminergic network in Nasonia.

To accomplish this, we first described the dopaminergic clusters in Nasonia brains, providing examples from $N$. vitripennis. In total, we identified 9 distinct clusters, two of which we consider subclusters. The two largest clusters, D2 and D3, can likely be further subdivided but we were unable to reliably do so in this study.

We then compared the number of cell bodies in these dopaminergic clusters between the species $N$. vitripennis and $N$. giraulti, as well as between normal- and small-sized $N$. vitripennis. We found significant differences in dopaminergic clusters that varied between the two different comparisons. In the interspecific comparison we found different cell numbers in clusters D2 and D4, whereas in the intraspecific comparison, differences were found in the D5 and D7 clusters.

\section{Comparison with other insects}

We present a comparison of the identified Nasonia clusters with those of three other species: the honey bee A. mellifera, the fruit fly $D$. melanogaster and the egg parasitoid T. evanescens. This comparison (Table 2) is based mainly on the location of these clusters in the cell body rind relative to landmark neuropils. For example, both the PAM cluster in the fruit fly and the $\mathrm{C} 1$ and $\mathrm{C} 2$ clusters in the honey bee are located at the anterior side of the brain, between the antennal lobe and the distal end of the vertical lobe of the mushroom body; a cluster found at this location in the Nasonia brain may be homologous to these clusters. Better comparisons can be made based on the projection patterns of the dopaminergic neurons in the clusters; unfortunately, our staining method targets all dopaminergic cells, which leads to many fibers crossing or running in parallel. The varicose nature of the immunolabeling further restricts the tracing of these fibers. The proposed homologies must therefore be considered to be strictly speculative and a potential starting point for further investigation.

Comparing dopamine-like immunoreactivity in Nasonia with these other species results in several interesting observations. First is the diversity of dopaminergic clusters between the compared species. Although we were able to make global comparisons, the location of these clusters does not appear to be very conserved. The clusters located around the subesophageal zone, in particular, seem quite divergent. Multiple small clusters have been described for the fruit fly (i.e., cell groups 8-15 in Nässel and Elekes 1992), the honey bee (i.e., S1-7 in Tedjakumala et al. 2017) and several in T. evanescens (i.e., DA-4, DA-7 in Van der Woude and
Table 1 Mean number of cells per cluster for the three groups of Nasonia studied. $N=$ number of hemispheres counted per cluster. Standard deviation for clusters for which no variation in cell body number was counted is labeled as n.a. MAX indicates the sum of the maximal amount of cells counted per cluster

\begin{tabular}{|c|c|c|c|c|c|c|c|c|c|c|c|c|c|c|c|}
\hline & & \multicolumn{4}{|c|}{ Nasonia giraulti } & \multicolumn{4}{|c|}{$N$. vitripennis (normal) } & \multicolumn{4}{|c|}{ N. vitripennis (small) } & & \\
\hline & & \multicolumn{3}{|c|}{ Number of cells } & \multirow[t]{2}{*}{$N=$} & \multicolumn{3}{|c|}{ Number of cells } & \multirow[t]{2}{*}{$N=$} & \multicolumn{3}{|c|}{ Number of cells } & \multirow[t]{2}{*}{$N=$} & & \\
\hline & & Mean & \pm & SD & & Mean & \pm & SD & & Mean & \pm & SD & & & \\
\hline \multirow[t]{10}{*}{ Cluster } & D1a & 3.43 & \pm & 0.82 & 14 & 3.77 & \pm & 0.70 & 13 & 3.11 & \pm & 0.74 & 9 & D1a & \multirow[t]{10}{*}{ Cluster } \\
\hline & D1b & 2.00 & & n.a. & 4 & 2.00 & & n.a. & 4 & 2.00 & & n.a. & 3 & $\mathrm{D} 1 \mathrm{~b}$ & \\
\hline & D2 & 47.08 & \pm & 11.15 & 13 & 59.85 & \pm & 11.11 & 13 & 50.88 & \pm & 13.13 & 8 & D2 & \\
\hline & D3 & 42.07 & \pm & 5.24 & 14 & 42.55 & \pm & 7.14 & 11 & 44.25 & \pm & 9.12 & 8 & D3 & \\
\hline & $\mathrm{D} 4 \mathrm{a}$ & 4.58 & \pm & 0.95 & 12 & 5.77 & \pm & 0.97 & 13 & 5.56 & \pm & 0.68 & 9 & $\mathrm{D} 4 \mathrm{a}$ & \\
\hline & $\mathrm{D} 4 \mathrm{~b}$ & 7.67 & \pm & 2.05 & 6 & 5.88 & \pm & 1.27 & 8 & 5.50 & \pm & 1.12 & 4 & $\mathrm{D} 4 \mathrm{~b}$ & \\
\hline & D5 & 3.14 & \pm & 1.64 & 7 & 3.75 & \pm & 1.20 & 8 & 2.38 & \pm & 0.70 & 8 & D5 & \\
\hline & D6 & 3.00 & & n.a. & 10 & 3.00 & & n.a. & 8 & 2.78 & \pm & 0.63 & 9 & D6 & \\
\hline & D7 & 1.33 & \pm & 0.47 & 3 & 2.00 & & n.a. & 3 & 1.00 & & n.a. & 4 & D7 & \\
\hline & MAX & 155 & & & & 169 & & & & 162 & & & & MAX & \\
\hline
\end{tabular}


Fig. 5 DA-L-IR cell bodies in brains of normal-sized $N$. vitripennis and $N$. giraulti. a Clusters D1, D4-D7. b Clusters D2 and D3 given separately, due to the relative abundance of cell bodies in these clusters. Bars depict average number of cell \pm standard deviation, dots depict raw counts. $* p<0.05$ (Wilcoxon)
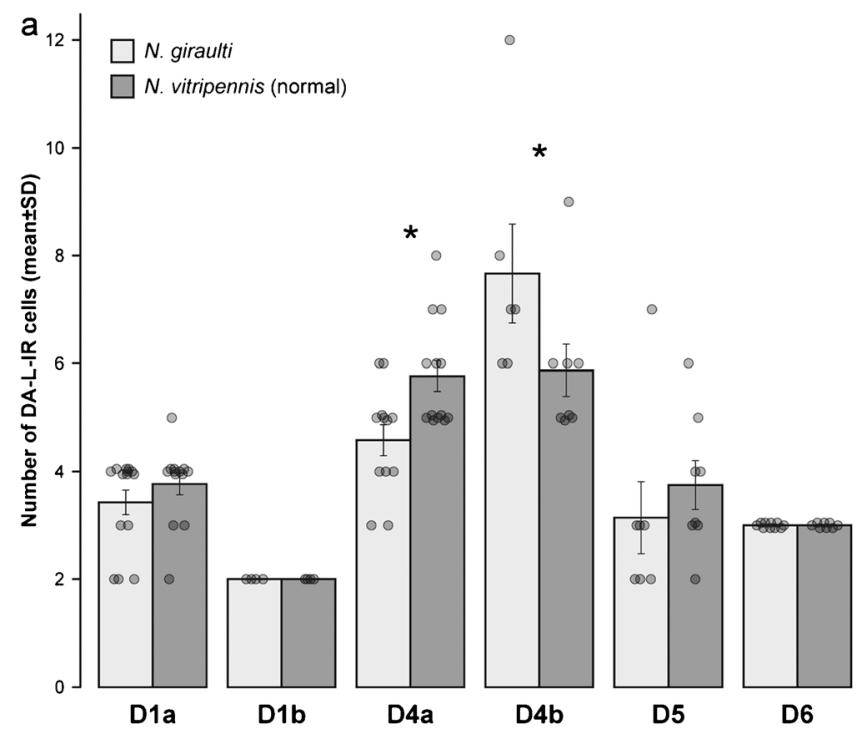

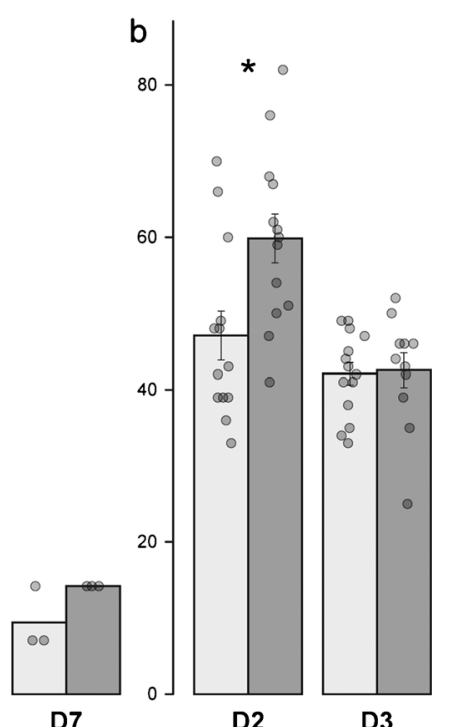

Smid 2017a) but in the Nasonia species studied here, we only found a few clusters with a low number of cells.

The relative abundance of cell bodies per cluster follows the same pattern in Nasonia, A. mellifera and D. melanogaster; the clusters that may correspond to D2 and D3 are most abundant. With a low number of cells in each cluster, T. evanescens appears to be the outlier. This may be an adaptation to the miniaturization in this tiny egg parasitoid (van der Woude and Smid 2017a).

We did not observe innervation of the mushroom body calyx or the optic lobes and only low levels of innervation of the antennal lobes. This is comparable to results in the other small parasitoid $T$. evanescens but not with A. mellifera and adult $D$. melanogaster, where innervation of these neuropils was clearly visible, albeit to a lesser extent than in the rest of the brain. Based on our current results, we cannot attribute the apparent lack of dopaminergic innervation in the calyx to a functional difference or to differences in methodology, as it has been shown that choosing the method to detect dopaminergic cells is a non-trivial matter. In A. mellifera, different results were obtained with an immunostaining against tyrosine hydroxylase (TH; the rate-limiting enzyme in dopamine synthesis; Tedjakumala et al. 2017) or against dopamine (Schäfer and Rehder 1989); likewise, Mao and Davis (2009) found different results by making use of the GAL4/UAS system or anti-TH staining. It could be that the apparent lack of an equivalent cluster to the D2/C1,2/PAM clusters, could be related to the specificity or sensitivity of the anti-dopamine antibody. On the other hand, there is no innervation of the MB calyx by dopaminergic neurons in the D. melanogaster larva (Eichler et al. 2017), whereas these larvae are fully capable of memory formation (as reviewed by Widmann et al. 2018). Whether a
Fig. 6 DA-L-IR cell bodies in brains of normal- and small-sized N. vitripennis. a Clusters D1, D4 D7. b Clusters D2 and D3 given separately, due to the relative abundance of cell bodies in these clusters. Bars depict average number of cell \pm standard deviation, dots depict raw counts. $* p<0.05$ (Wilcoxon)
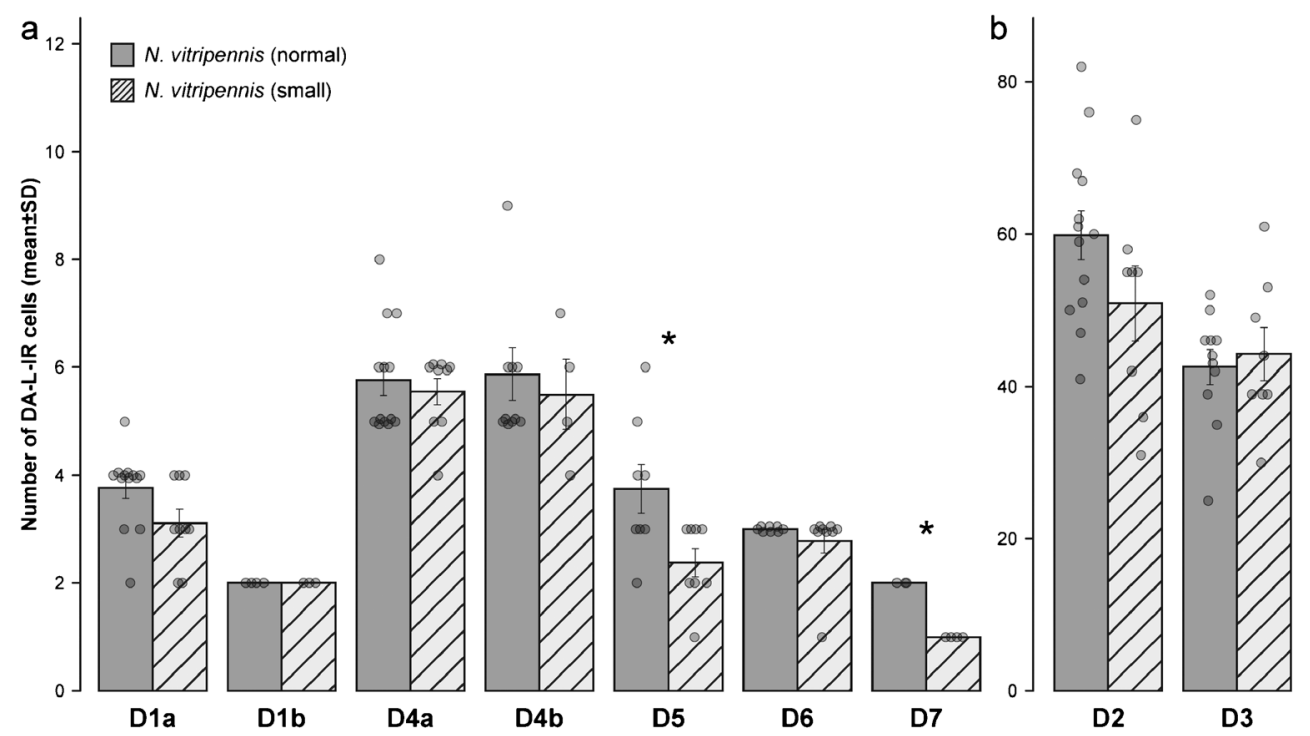
Table 2 A comparison of dopaminergic clusters in normal-sized $N$. vitripennis (DA-L-IR, this study), A. mellifera (tyrosine hydroxylase (TH)-IR, Tedjakumala et al. 2017), D. melanogaster (TH-IR, TH-Gal4, Mao and Davis 2009 and TH-IR, Nässel and Elekes 1992 for SEZ clusters) and T. evanescens (DA-L-IR, van der Woude and Smid 2017a). Clusters are grouped by location of somata in the cell body rind and, when possible, projection patterns. This grouping is tentative and does not guarantee homology between the clusters

\begin{tabular}{|c|c|c|c|c|c|c|c|}
\hline \multicolumn{2}{|c|}{ N. vitripennis } & \multicolumn{2}{|l|}{ A. mellifera } & \multicolumn{2}{|c|}{ D. melanogaster } & \multicolumn{2}{|c|}{ T. evanescens } \\
\hline Cluster & Cells & Cluster & Cells & Cluster & Cells & Cluster & Cells \\
\hline $\begin{array}{l}\text { D1a } \\
\text { D1b }\end{array}$ & $\begin{array}{l}3.77 \\
2.00\end{array}$ & ST/AOTU & $2-3$ & PAL & 5 & DA-1 & 2.6 \\
\hline D2 & 59.85 & $\begin{array}{l}\mathrm{C} 1 * \\
\mathrm{C} 2 *\end{array}$ & $\begin{array}{l}75 \\
75\end{array}$ & PAM* $^{*}$ & $\sim 100$ & DA-3? & 2.7 \\
\hline D3 & 42.55 & $\mathrm{C} 3 *$ & 140 & $\begin{array}{l}\text { PPL1* } \\
\text { PPL2c } \\
\text { PPL4 } \\
\text { PPL5 }\end{array}$ & $\begin{array}{l}12 \\
4.6 \\
0.91 \\
0.54\end{array}$ & DA-5b & 3.9 \\
\hline $\begin{array}{l}\mathrm{D} 4 \mathrm{a} \\
\mathrm{D} 4 \mathrm{~b}\end{array}$ & $\begin{array}{l}5.77 \\
5.88\end{array}$ & SP & $15-20$ & $\begin{array}{l}\text { PPM1 } \\
\text { PPM2 }\end{array}$ & $\begin{array}{l}5.46 \\
8.07\end{array}$ & DA-6 & 3.2 \\
\hline D5 & 3.75 & SL & $5-8$ & PPL2ab* & 6.1 & DA-2 & 2.3 \\
\hline D6 & 3.00 & S1-2 & 4 & SA1, SP1-2 & 1,2 & DA-7 & 1.4 \\
\hline D7 & 2.00 & S7-8 & $2-4$ & SVP1 & 1 & DA-4 & 2.5 \\
\hline
\end{tabular}

*Confirmed innervation of the mushroom bodies

lack of dopaminergic innervation in the calyx is a trait common to other small Hymenoptera besides Trichogramma and Nasonia is a topic that warrants further study.

\section{Species differences in Nasonia}

The strains of Nasonia vitripennis and $N$. giraulti that we used in our study differ in their memory dynamics, with $N$. giraulti requiring multiple, spaced experiences before consolidating protein synthesis-dependent long-term memory (Hoedjes et al. 2012). A previous study comparing the octopaminergic network in these wasps was unable to show differences in this system (Haverkamp and Smid 2014). Contrary to that study, we show here that normal-sized $N$. vitripennis and normalsized $N$. giraulti do indeed differ in their dopaminergic network. Our analysis revealed that $N$. vitripennis had more cells in the D2 and D4a clusters but fewer in the D4b cluster.

As discussed above, the position and numbers of neurons in the D2 cluster corresponds to mushroom body-projecting clusters in other species, such as the PAM cluster in D. melanogaster and the $\mathrm{C} 1$ and $\mathrm{C} 2$ clusters in A. mellifera. This PAM cluster contains both cells associated with aversive and reward signaling (Waddell 2013). The structural variation between the two Nasonia species that differ in memory formation may be interesting for further analysis.

The D4 clusters are most similar to clusters that project to and around the central complex in A. mellifera (SP cluster) and D. melanogaster (PPM1/2 clusters). Dopaminergic innervation in the central complex is important for motor control (reviewed by Pfeiffer and Homberg 2014) but behavioral implications of $N$. vitripennis having more cells in $\mathrm{D} 4 \mathrm{a}$ and less in D4b are unclear. It may be that our arbitrary distinction of these subclusters based on distance is incorrect and that the effects we found cancel out.

Our comparison of dopaminergic clusters, the previous comparison of octopaminergic clusters (Haverkamp and Smid 2014) and the difference in memory dynamics that instigated these comparisons (Hoedjes et al. 2012) were all described for the isogenic AsymCx and R2VU(x) strains of $N$. vitripennis and $N$. giraulti, respectively. As genetic variation may influence $N$. vitripennis and $N$. giraulti memory dynamics (Koppik et al. 2015; Liefting et al. 2018), the use of isogenic lines helps to minimize variation and pinpoint potential causalities. Both the AsymCx and R2VU(x) strains have a sequenced genome (Werren et al. 2010) that may aid further characterization of dopaminergic cluster size. Of particular interest for further investigation is that this difference in memory dynamics has been introgressed by hybridization of these strains, yielding $N$. giraulti-like memory in an $N$. vitripennis genetic background (Hoedjes et al. 2014).

\section{Size-related differences in N. vitripennis}

To our knowledge, this is the second study to compare the dopaminergic system in relation to body size in insects (the first being a comparison of the highly diverse body sizes in T. evanescens (van der Woude and Smid 2017b)). Despite the almost threefold differences in absolute brain volume between normal- and small-sized N. vitripennis (Groothuis and Smid 2017), we observed no difference in the total number of dopaminergic cells. This is comparable to the effects of extreme (fivefold) brain scaling in $T$. evanescens, where no difference 
in numbers of dopaminergic neurons was found (van der Woude and Smid 2017b). In T. evanescens these neurons are significantly smaller in the smallest individuals. Though we intended to analyze similar measurements in $N$. vitripennis, we could not reliably label the entire cell body in every instance. It may be that dopamine is not sufficiently present throughout the cell bodies of $N$. vitripennis, restricting the use of an anti-dopamine stain.

In contrast to the $T$. evanescens study, we did find clusterspecific differences between $N$. vitripennis of different size. Small individuals had fewer cells in the D5 and in the D7 cluster. The D5 cluster could be homologous to the PPL2ab cluster of D. melanogaster based on the position of the cell bodies, which is known to project to the mushroom body calyx and the lateral horn (Mao and Davis 2009). These neuropils are known to be involved with learned and naïve odor preferences, respectively (Strutz et al. 2014). However, the exact function of the neurons in the PPL2ab cluster have, to our knowledge, not been described in other species and as mentioned earlier, we did not reveal any dopaminergic innervation in the calyx. We cannot speculate on the homology and functional role of the D7 cluster, or could we observe its projections. We note, however, that the D7 cluster could only be counted in a small number of brains in both size classes (see Table 1).

\section{Future prospects}

Owing to hymenopteran male haploid genetics, introgression of traits that differ between strains and species is relatively easy in Nasonia (Hoedjes et al. 2014; Werren et al. 2016). As mentioned above, the memory dynamics central to this study have been introgressed, yielding $N$. giraulti-like memory linked to two specific quantitative trait loci in a predominantly $N$. vitripennis genetic background (Hoedjes et al. 2014). Future lines of research may focus on the dopaminergic networks of the resulting interspecies hybrid wasps. If the differences found in our work indeed explain the difference in memory dynamics, these future studies may find similar differences in the dopaminergic system of introgressed hybrid lines, such as $N$. giraulti-like numbers of D2 cells.

Furthermore, the characterization of dopaminergic clusters and their innervations may be improved by the development of better cell type-specific detection methods that were not available at the time of this study, such as CRISPR/Cas9 (Lynch 2015; Li et al. 2017).

\section{Data accessibility}

An example of a three-dimensional confocal microscopy stack has been made available at the Insect Brain Database: https:// hdl.handle.net/20.500.12158/SIN-0000022.2
Acknowledgments The authors thank M. Boumans for preliminary experimental work, M.E. Huigens for fruitful discussions, M. Dicke for help in manuscript preparation and H. Schipper (Wageningen University \& Research, Experimental Zoology) for the use of the confocal laser scanning microscope. Two anonymous reviewers made valuable suggestions to improve the manuscript.

Funding information This work was supported by NWO Open Competition grant 820.01.012.

Compliance with ethical standards All applicable international, national and/or institutional guidelines for the care and use of animals were followed.

Conflict of interest The authors declare that they have no conflict of interest.

Open Access This article is distributed under the terms of the Creative Commons Attribution 4.0 International License (http:// creativecommons.org/licenses/by/4.0/), which permits unrestricted use, distribution, and reproduction in any medium, provided you give appropriate credit to the original author(s) and the source, provide a link to the Creative Commons license, and indicate if changes were made.

\section{References}

Aso Y, Herb A, Ogueta M et al (2012) Three dopamine pathways induce aversive odor memories with different stability. PLoS Genet 8: e1002768

Burke CJ, Huetteroth W, Owald D et al (2012) Layered reward signalling through octopamine and dopamine in Drosophila. Nature 492:433437

Claridge-Chang A, Roorda RD, Vrontou E et al (2009) Writing memories with light-addressable reinforcement circuitry. Cell 139:405-415

Cognigni P, Felsenberg J, Waddell S (2018) Do the right thing: neural network mechanisms of memory formation, expression and update in Drosophila. Curr Opin Neurobiol 49:51-58

Das G, Lin S, Waddell S (2016) Remembering components of food in Drosophila. Front Integr Neurosci 10:4

Eichler K, Li F, Litwin-Kumar A et al (2017) The complete connectome of a learning and memory centre in an insect brain. Nature 548:175182

Groothuis J, Smid HM (2017) Nasonia parasitic wasps escape from Haller's rule by diphasic, partially isometric brain-body size scaling and selective neuropil adaptations. Brain Behav Evol 90:243-254

Hammer M (1993) An identified neuron mediates the unconditioned stimulus in associative olfactory learning in honeybees. Nature 366:59-63

Hammer M, Menzel R (1998) Multiple sites of associative odor learning as revealed by local brain microinjections of octopamine in honeybees. Learn Mem 5:146-156

Haverkamp A, Smid HM (2014) Octopamine-like immunoreactive neurons in the brain and subesophageal ganglion of the parasitic wasps Nasonia vitripennis and N. giraulti. Cell Tissue Res 358:313-329

Hoedjes KM, Smid HM (2014) Natural variation in long-term memory formation among Nasonia parasitic wasp species. Behav Process $105: 40-45$

Hoedjes KM, Steidle JLM, Werren JH et al (2012) High-throughput olfactory conditioning and memory retention test show variation in Nasonia parasitic wasps. Genes Brain Behav 11:879-887 
Hoedjes KM, Smid HM, Vet LEM, Werren JH (2014) Introgression study reveals two quantitative trait loci involved in interspecific variation in memory retention among Nasonia wasp species. Heredity 113: $542-550$

Ito K, Shinomiya K, Ito M et al (2014) A systematic nomenclature for the insect brain. Neuron 81:755-765

Kaun KR, Rothenfluh A (2017) Dopaminergic rules of engagement for memory in Drosophila. Curr Opin Neurobiol 43:56-62

Koppik M, Hoffmeister TS, Brunkhorst S et al (2015) Intraspecific variability in associative learning in the parasitic wasp Nasonia vitripennis. Anim Cogn 18:593-604

Krashes MJ, DasGupta S, Vreede A et al (2009) A neural circuit mechanism integrating motivational state with memory expression in Drosophila. Cell 139:416-427

Li M, Au LYC, Douglah D et al (2017) Generation of heritable germline mutations in the jewel wasp Nasonia vitripennis using CRISPR/ Cas9. Sci Rep 7:901

Liefting M, Hoedjes KM, Lann CL et al (2018) Selection for associative learning of color stimuli reveals correlated evolution of this learning ability across multiple stimuli and rewards. Evolution 72:14491459

Lin S, Owald D, Chandra V et al (2014) Neural correlates of water reward in thirsty Drosophila. Nat Neurosci 17:1536-1542

Liu C, Placais PY, Yamagata N et al (2012) A subset of dopamine neurons signals reward for odour memory in Drosophila. Nature 488:512516

Lynch JA (2015) The expanding genetic toolbox of the wasp Nasonia vitripennis and its relatives. Genetics 199:897-904

Mao ZM, Davis RL (2009) Eight different types of dopaminergic neurons innervate the Drosophila mushroom body neuropil: anatomical and physiological heterogeneity. Front Neural Circuit 3:5

Nässel DR, Elekes K (1992) Aminergic neurons in the brain of blowflies and Drosophila - dopamine-immunoreactive and tyrosine hydroxylase-immunoreactive neurons and their relationship with putative histaminergic neurons. Cell Tissue Res 267:147-167

Pfeiffer K, Homberg U (2014) Organization and functional roles of the central complex in the insect brain. Annu Rev Entomol 59:165-184

Rago A, Gilbert DG, Choi JH et al (2016) OGS2: genome re-annotation of the jewel wasp Nasonia vitripennis. BMC Genomics 17:678

Rohwedder A, Wenz NL, Stehle B et al (2016) Four individually identified paired dopamine neurons signal reward in larval Drosophila. Curr Biol 26:661-669

Schäfer S, Rehder V (1989) Dopamine-like immunoreactivity in the brain and suboesophageal ganglion of the honeybee. J Comp Neurol 280: $43-58$
Schürmann FW, Elekes K, Geffard M (1989) Dopamine-like immunoreactivity in the bee brain. Cell Tissue Res 256:399-410

Schurmann D, Sommer C, Schinko APB et al (2012) Demonstration of long-term memory in the parasitic wasp Nasonia vitripennis. Entomol Exp Appl 143:199-206

Schwaerzel M, Monastirioti M, Scholz H et al (2003) Dopamine and octopamine differentiate between aversive and appetitive olfactory memories in Drosophila. J Neurosci 23:10495-10502

Smid HM, Vet LEM (2016) The complexity of learning, memory and neural processes in an evolutionary ecological context. Curr Opin Insect Sci 15:61-69

Strutz A, Soelter J, Baschwitz A et al (2014) Decoding odor quality and intensity in the Drosophila brain. eLife 3:e04147

Tedjakumala SR, Rouquette J, Boizeau ML et al (2017) A tyrosinehydroxylase characterization of dopaminergic neurons in the honey bee brain. Front Syst Neurosci 11:47

Vergoz V, Roussel E, Sandoz J-C, Giurfa M (2007) Aversive learning in honeybees revealed by the olfactory conditioning of the sting extension reflex. PLoS One 2:e288

Waddell S (2013) Reinforcement signalling in Drosophila; dopamine does it all after all. Curr Opin Neurobiol 23:324-329

Werren JH, Loehlin DW (2009) The parasitoid wasp Nasonia: an emerging model system with haploid male genetics. Cold Spring Harb Protoc 4:pdb emo134

Werren JH, Richards S, Desjardins CA et al (2010) Functional and evolutionary insights from the genomes of three parasitoid Nasonia species. Science 327:343-348

Werren JH, Cohen LB, Gadau J et al (2016) Dissection of the complex genetic basis of craniofacial anomalies using haploid genetics and interspecies hybrids in Nasonia wasps. Dev Biol 415:391-405

Widmann A, Eichler K, Selcho M et al (2018) Odor-taste learning in Drosophila larvae. J Insect Physiol 106:47-54

van der Woude E, Smid HM (2017a) Maximized complexity in miniaturized brains: morphology and distribution of octopaminergic, dopaminergic and serotonergic neurons in the parasitic wasp, Trichogramma evanescens. Cell Tissue Res 369:477-496

van der Woude E, Smid HM (2017b) Effects of isometric brain-body size scaling on the complexity of monoaminergic neurons in a minute parasitic wasp. Brain Behav Evol 89:185-194

van der Woude E, Huigens ME, Smid HM (2018) Differential effects of brain size on memory performance in parasitic wasps. Anim Behav $141: 57-66$

Publisher's note Springer Nature remains neutral with regard to jurisdictional claims in published maps and institutional affiliations. 